\title{
Self calibration of sensorless adaptive optical microscopes
}

\author{
Anisha Thayil \\ Martin J. Booth \\ martin.booth@eng.ox.ac.uk
}

\begin{abstract}
Department of Engineering Science, University of Oxford, Parks Road, Oxford OX1 3PJ, United Kingdom

Department of Engineering Science, University of Oxford, Parks Road, Oxford, OX1 3PJ, United Kingdom
\end{abstract}

We present a self-calibrating scheme for microscopes using model-based wavefront sensorless adaptive optics. Unlike previous methods, this scheme permits the calibration of system aberration modes without the need for a separate wavefront sensor or interferometer. Basis modes are derived from the deformable mirror influence functions and an image cross-correlation method is used to remove image displacement effects from these modes. Image based measurements are used to derive an optimum modal representation from the displacement-free basis modes. These new modes are insensitive to system misalignments and the shape of the illumination profile. We demonstrate the effectiveness and robustness of these optimal modes in a third harmonic generation (THG) microscope. [D0I: http: //dx.doi.org/10.2971/jeos.2011.11045]

Keywords: Adaptive optics, aberrations, microscopy

\section{INTRODUCTION}

Aberrations frequently affect the performance of high resolution microscopes. In order to overcome this problem, adaptive optics $(\mathrm{AO})$ techniques have been introduced in a range of different microscope modalities. These AO systems employ an adaptive element, such as a deformable mirror (DM) or spatial light modulator, to correct the specimen and system-induced aberrations. Unlike in conventional adaptive optics systems, most of the AO microscope implementations have been "sensorless", employing indirect wavefront measurements rather than wavefront sensors. Modal methods of sensorless AO provide an efficient method of indirect wavefront measurement, but require careful calibration of the adaptive element to function effectively, particularly when using a DM; this calibration encodes the control signals that generate aberration modes from a suitable basis set, such as the Zernike polynomials. For this reason, systems have incorporated interferometers or wavefront sensors, which required increased complexity in the overall optical design and operation. The operation of these systems is also sensitive to the alignment of the adaptive element relative to the pupil of the objective lens [1].

The optical layout of these AO microscopes could be considerably simplified if the sensor or interferometer were removed. Further benefit would be obtained by using a calibration scheme that is insensitive to misalignments. In this paper, we propose a fully empirical determination of aberration modes that can be implemented in any optical microscope and does not require a wave front sensor. We demonstrate this scheme in an adaptive third harmonic generation (THG) microscope and show its effectiveness in the presence of system misalignments.

\section{Principles of sensorless adaptive optics}

We outline in this section the principles of model-based sensorless AO schemes for use in microscopes. In particular, we explain the importance of the choice of modal expansion for the aberration representation and the relationship between image shifts and certain aberration modes.

\subsection{Functional representation of aberrations}

The DM is controlled by a set of signals that drive the individual actuators that deform the mirror surface. This produces a limited range of shapes that are determined by the mechanical properties of the mirror and the actuator arrangement. The influence function of an actuator is defined as the DM shape produced when a unit signal is applied to that actuator. We assume that the DM operates in a regime where linearised control signals can be used. This means that the overall shape of the DM is determined by the linear superposition of the actuator influence functions. This is a reasonable approximation for many practical systems, particularly for small aberration amplitudes. The DM aberration can then be expressed as

$$
\Phi(\mathbf{r})=\sum_{i=1}^{N} c_{i} \psi_{i}(\mathbf{r})
$$

where $N$ is the number of actuators, $c_{i}$ and $\psi_{i}$ are respectively the control signal and the influence function for the $i$ th actuator, and $\mathbf{r}$ is the position vector in the pupil.

The set of influence functions forms a basis that can represent any DM shape. However, it is usually desirable to control 
the DM using a different set of basis modes that have particular mathematical characteristics, such as orthogonality. In this case, we can express the aberration as a combination of $M$ modes:

$$
\Phi(\mathbf{r})=\sum_{i=1}^{M} a_{i} X_{i}(\mathbf{r})
$$

where $X_{i}$ is the $i$ th mode with coefficient $a_{i}$. The control signals are related to the modal coefficients via a linear transformation, represented by the matrix-vector equation

$$
\mathbf{c}=\mathbf{B a}
$$

where $\mathbf{B}$ is the $N \times M$ transformation matrix and $a_{i}$ and $c_{i}$ comprise respectively the elements of the vectors a and c. For mathematical convenience, sets of analytic functions such as Zernike polynomials are frequently used as the basis modes. As these modes do not perfectly describe the mirror deformations, the representations of Eqs. 1 and 2 are not equivalent and describe different function spaces. Instead of analytical modes, one can derive sets of deformation modes that are linear combinations the influence functions. These are advantageous compared to analytic modes as they match perfectly the actual deformations of the mirror. In this case, the number of modes is exactly the same as the number of actuators $(M=N)$ and the square matrix B describes a (usually non-singular) linear transformation.

In model-based sensorless $\mathrm{AO}$ systems, the modes are chosen to exhibit properties that enable optimum performance of the AO scheme. The derivation of the optimum modes from the basis sets is outlined in the following sections.

\subsection{Aberrations and displacements in three-dimensional imaging systems}

In the context of imaging systems, aberrations modes can be separated into three types: those that have no effect on images, those that cause image displacement, and those that affect image quality. The simplest aberration is the piston mode - a constant phase offset. In most microscopes, piston has no effect on the imaging process or the AO system. Hence, it is frequently neglected in analysis. Throughout this paper, for simplicity, all aberrations are assumed to have zero mean phase and hence zero piston component.

The tip and tilt modes consist of linear phase gradients in orthogonal directions across the pupil and correspond to lateral translations in the image plane. There also exists a defocus mode that is equivalent to a refocusing of the imaging system. In the paraxial approximation, the defocus mode varies quadratically with radius; in high numerical aperture (NA) system, it is a higher order radial function. The three modes tip, tilt and defocus represent image translations or distortions in three dimensions; they do not however affect the resolution or contrast of the image. From here-on, we shall refer collectively to the tip, tilt and defocus modes as "displacement modes". All other aberration modes affect the resolution and contrast of images.

In three-dimensional imaging $\mathrm{AO}$ systems, it is important to avoid image displacements during the correction process. To achieve this, displacement modes should be excluded from the AO scheme. Moreover, the other aberration modes should not contain any component of the displacement modes, as their inclusion would induce image shifts during the aberration correction process, which could in turn affect aberration measurements. By excluding displacement modes, one can ensure that only the aberration components affecting image quality will influence the measurements. Furthermore, the exclusion of displacement modes reduces the total DM surface distortion, thus reduces the possibility of actuator saturation during operation.

\subsection{Optimum modes for model-based sensorless adaptive optics}

A sensorless adaptive optics scheme is based around the optimisation of a chosen metric, such as image intensity. This metric should be chosen to enable an efficient optimisation process. One approach is to choose a metric with a well-defined parabolic maximum, where the maximum metric value corresponds to the best imaging conditions. This is usually equivalent to zero aberration. It has been shown in $[2,3]$ that for any choice of metric with this property and any set of basis modes $X_{i}$, a new set of optimal modes $Y_{i}$ can be derived, which enable the metric to be expressed in separable quadratic form:

$$
M \approx q\left(1-\sum_{i=1}^{N} \beta_{i} b_{i}^{2}\right)
$$

where $q$ and $\beta_{i}$ are constants and $b_{i}$ are the coefficients of the new modes. As the different modal coefficients appear separately in the expansion of $M$, each mode can be optimised independently using a one-variable quadratic maximisation algorithm without explicit knowledge of the other variables. It has been shown [3] that the required quadratic characteristic is linked to the orthogonality of the modes $Y_{i}$, where orthogonality is defined by a particular inner product (IP). In principle, the IP can be derived mathematically from the Taylor expansion of the metric with respect to the modal coefficients. However, the complexity of the mathematics describing the imaging process means that simple expressions for the inner product are not readily obtained for many microscopes. Similarly, conventional analytic modal sets, such as the Zernike polynomials, may not have the required mathematical properties.

Generally, it is necessary to derive new sets of modes for a particular application to ensure optimal performance of the sensorless system. Three different methods that have been used to obtain optimum modes in these systems - analytical, numerical and empirical - are explained below.

1. Analytical: In some situations, it is possible to define a set of analytic functions that are orthogonal with respect to the inner product. This method has been employed, for example, using Zernike modes in a focussing system [4] or Lukosz modes in a focussing system [5] or in an incoherent microscope using image low spatial frequency content as the metric [6]. This analytical approach is of relatively limited application, as there are few sets of 
known analytic modes that could be matched to any particular adaptive system.

2. Numerical: If the functional form of the inner product is known, for example from the Taylor expansion of the metric function, then the optimum orthogonal modes can be obtained numerically. As a starting point, one selects a suitable set of modes as a basis set (e.g. a subset of loworder Zernike polynomials). The optimum modes are then constructed from the basis set using an orthogonalisation process based around the inner product. This has been shown in structured illumination and two-photon fluorescence microscopes [3, 2].

3. Empirical: If the functional description of the inner product is not available, the basis modes can be orthogonalised using an empirical process in which the form of the metric function is determined from image measurements. A sequence of images is acquired with different bias aberrations applied by the adaptive element. The corresponding metric measurements map out the shape of the metric function in the vicinity of the paraboloidal peak. The new modes are obtained as a linear combination of the basis modes by finding the coordinate system that aligns the primary axes of the paraboloidal maximum with the coordinate axes. This approach has been demonstrated in structured illumination, two-photon and harmonic generation microscopes $[3,2,7]$.

In each of these three approaches, it is essential that the displacement modes are excluded from the basis modes, to avoid the problems associated with image shifts. In method 1, the displacements modes are excluded simply by removing the corresponding modes from the analytic set. In implementations of methods 2 and 3, the displacement modes have been removed from the basis set in advance of the orthogonalisation process. In each case, the derivation of the modes required the direct measurement of the DM introduced phase using a wavefront sensor or interferometer. These measurements provided the information required to calculate and remove the displacement modes from the basis set.

\subsection{The correction procedure}

The modal aberration correction scheme is based upon the sequential correction of a suitable set of $N$ basis modes through maximisation of a chosen image quality metric, such as the total image intensity. The maximisation is implemented by intentionally introducing predetermined aberrations, or biases, into the system using the DM. In this paper, we use the following procedure. First, a positive bias $+b Y_{i}$ of the measured mode $Y_{i}$ is added, where $b$ was the bias amplitude, and an image is acquired; from this image the metric value $M_{+}$is calculated. Then a negative bias of the same mode $-b Y_{i}$ is applied, providing the metric value $M_{-}$. Additionally, the metric value $M_{0}$ is calculated with no bias aberration. The bias amplitude $b$ is chosen to have a suitable value, which is typically less than the half width at half maximum of the response curve of $M$ for that mode being measured. The correction aberration is then found through a maximisation procedure defined as:

$$
a_{\text {corr }}=\frac{b\left(g_{+}-g_{-}\right)}{2 g_{+}-4 g_{0}+2 g_{-}},
$$

where $g_{+}, g_{-}$and $g_{0}$ are the reciprocal of the metric values $M_{+}, M_{-}$, and $M_{0}$ respectively. The measurement and correction cycle is then repeated for each of the $N$ modes of interest. Correction of $N$ modes require $2 N+1$ measurements in total. This cycle is then repeated to test whether convergence is complete.

\section{Fully empirical mode calibration}

\subsection{Limitations of previous sensorless adaptive optics schemes}

The specification of a sensorless $\mathrm{AO}$ scheme, as outlined above, relies upon the accurate correspondence between the mathematical description of the aberration modes and their realisation in the optical system. In practice, there are various sources of error that can lead to discrepancies. There are two major sources of error that can affect sensorless AO systems: non-common-path errors and alignment errors. We now discuss the origin and effects of these errors.

Non-common path errors: The derivation of modes using the methods described above requires at some stage measurement of the influence functions. This can be implemented using a wavefront sensor or an interferometer. These measurements may include additional aberrations that are not encountered in the microscope optical path. This arises because the sensor must in practice use an optical path that is not common to the microscope imaging path. As a consequence, the influence function measurements may include systematic errors. When modes are obtained empirically, the effects of these errors are reduced: Although the initial influence functions are obtained using the sensor, the final modes are obtained via image-based measurements that rely only upon the microscope imaging path.

Alignment errors: It is essential that the active area of the adaptive element is correctly imaged on to the pupil of the objective lens and the sensor pupil. A slight offset in the lateral position of the objective pupil could lead to significant errors in the pupil phase, especially where regions from outside of the sensor pupil lie inside the objective pupil. Similar problems occur if the magnification between the pupils is incorrect. With such misalignments, even when the sensor detects that a mode is perfectly generated by the DM, the actual phase at the objective pupil could be considerably different. Significant experimental effort has been expended in ensuring that the correct pupil was used for the DM calibration. For example, in references $[3,2,8]$ the beam was passed through the objective lens, then reflected from a mirror back through the same lens in order to ensure the correct limiting aperture from the objective pupil was used at the sensor. This adds complexity to the system and can introduce additional non-common path aberrations from the extra optical components.

We propose to eliminate these errors by implementing a calibration procedure that is entirely sensorless and does not need 
a wavefront sensor or interferometer for the initial calibration. This removes the existing limitation of the empirical mode derivation method, which required measurement of the basis modes in order to remove any displacement components.

\subsection{Outline of the empirical calibration scheme}

The first step of proposed scheme is the calculation of influence functions using an approximate model of the DM mechanical response. Mirror deformation modes are then constructed from the set of influence functions. The next step is the removal of displacement mode components from the deformation modes.

Rather than starting with a mathematical description of displacement modes, we instead define the displacements in terms of the phenomenon of image shifts. In an ideal imaging system, this definition of the three displacement modes should correspond perfectly with the $\mathrm{x}, \mathrm{y}$ and $\mathrm{z}$ image shifts. These shifts are quantified by observation of the effects of each mode on a three-dimensional image stack. As the light forming the image follows exactly the path we need to correct, the method automatically corrects for any alignment errors and avoids any non-common path errors. The resulting displacement-free deformation modes form the basis set for the empirical derivation of the optimal modes.

The following sections detail the steps of this calibration scheme and its implementation in a practical microscope system. For this demonstration, we used an adaptive thirdharmonic generation (THG) microscope [8], although the method is applicable to all three-dimensional microscope systems. The system consisted of a Cr:Forsterite laser (DelMar Photonics Mavericks), which emitted $65 \mathrm{fs}$ pulses at a repetition rate of $76 \mathrm{MHz}$. The central wavelength was at $1230 \mathrm{~nm}$ and the output average power was about $200 \mathrm{~mW}$. Laser scanning was performed by a pair of galvanometric mirrors (VM1000, GSI Lumonics) which were imaged onto the deformable membrane mirror (MIRAO 52-e, Imagine Optic). The DM was then imaged onto the pupil plane of the objective lens (UApo/340 water immersion objective, $40 \times, \mathrm{NA}=1.15)$. The THG signal was collected in transconfiguration by an oil immersion condenser (NA=1.4) and was focussed onto a photomultiplier tube (PMT). A suitable bandpass filter was inserted before the PMT to filter out THG signal from the illumination light. Specimen scanning in the axial direction was performed by a piezo actuator attached to the sample stage.

\subsection{Derivation of basis modes}

Mirror deformation modes were chosen as the initial basis set. In order to derive these modes, we first obtained influence functions by solving the membrane equation, which describes the surface displacement of a perfect thin membrane in the presence of distributed forces [9]. Whilst not providing a full description of the mechanical properties of the MIRAO DM, this model is sufficient to provide close approximations to the actual influence functions. The set of eigenmodes

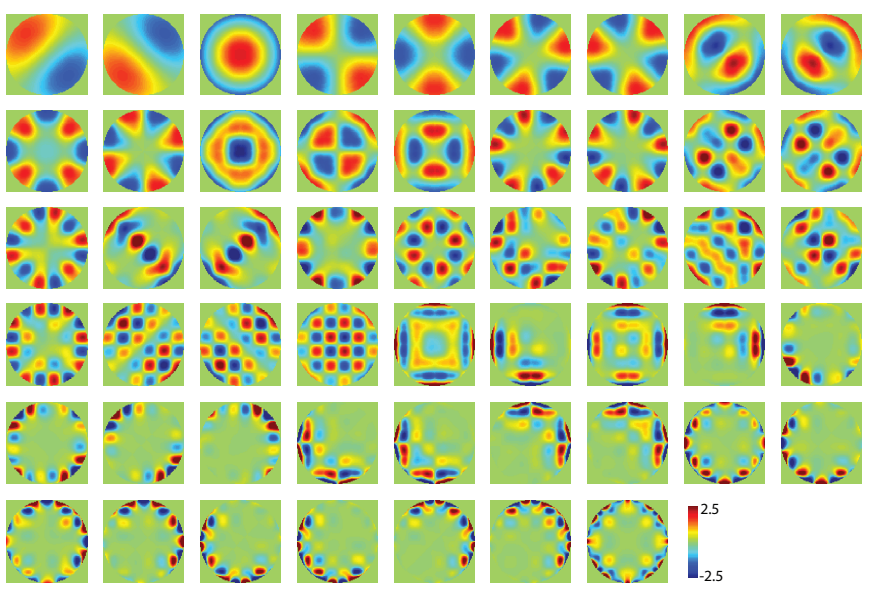

FIG. 1 Normalised plots of the approximate deformation modes of the MIRAO DM derived using the membrane equation. A subset consisting of the first 14 of these modes was used as the basis set for the derivation of optimum modes.

was obtained by orthogonalisation of the influence functions, performed using principal component analysis as outlined in [10]. This provided the set of control signals required to generate the basis modes on the DM and hence the control matrix B of Eq. 3. As an approximate mechanical model was used, the actual modes generated by the DM may not have been perfectly orthogonal. However, the modes did form a wellconditioned basis set that spanned the function space of the DM. As such, they formed a suitable basis for derivation of the optimum modes. The basis functions obtained in this way are denoted $X_{i}$. As the aim of this investigation was to omit a wavefront sensor from the microscope system, we do not present direct measurements of these modes, as produced by the DM. However, for the purposes of visualisation, Figure 1 shows the calculated shapes of the 52 basis modes derived for the MIRAO DM. As an approximate mechanical model was used, these shapes may differ slightly from the actual shape of the DM.

In principle, the influence functions themselves could be used as the basis set for the following procedure. However, it has been found in previous investigations that a modal basis, even if approximate, provides a better conditioned starting point for this procedure. This is because the properties of the modal basis set are closer than those of the influence functions to the orthogonal properties required for optimal operation of the correction scheme.

\subsection{Derivation of displacement-free optimal modes}

\subsubsection{Measurement of tip, tilt and defocus from image shifts}

The empirically derived displacement modes were obtained from the basis modes by the following process. A specimen consisting of glass beads (of approximate diameter $10 \mu \mathrm{m}$ ) suspended in gelatin was chosen due to its fine scale threedimensional structure. The DM was set such that all system aberrations were corrected and a 3D reference image stack $I_{0}(x, y, z)$ encompassing a single bead was acquired. A chosen amplitude $a$ of a basis mode $X_{i}$ was added to the DM and 
another image stack $I_{1}(x, y, z)$ was acquired. The relative displacement between the two stacks was calculated through image cross correlation. For the $x$ direction the correlation can be expressed as

$$
R_{x}\left(x^{\prime}\right)=\iiint_{x, y, z} I_{0}(x, y, z) I_{1}\left(x-x^{\prime}, y, z\right) \mathrm{d} x \mathrm{~d} y \mathrm{~d} z
$$

with similar expressions used for $y$ and $z$. The image shift was then obtained by finding the value of $x^{\prime}$ that maximised $R_{x}\left(x^{\prime}\right)$. This was repeated for a range of different mode amplitudes $a_{k}$, resulting in image shifts $x_{k}^{\prime}$. A linear fit to these data provided the gradient $s_{i}^{x}$, which quantified the amount of image shift in the $x$-direction per unit amplitude of basis mode $X_{i}$. Similar calculations were performed to obtain the equivalent gradients $s_{i}^{y}$ and $s_{i}^{z}$. This was repeated for each basis mode $X_{i}$ for $i=1$ to $N$.

Assuming that the effects of each mode add linearly, the total image shifts from an aberration $\sum_{i=1}^{N} a_{i} X_{i}$ can be derived from these measurements as

$$
s_{x}=\sum_{i=1}^{N} s_{i}^{x} a_{i}, s_{y}=\sum_{i=1}^{N} s_{i}^{y} a_{i}, s_{z}=\sum_{i=1}^{N} s_{i}^{z} a_{i} .
$$

Alternatively, this can be represented by the matrix equation

$$
\mathbf{s}=\mathbf{M a},
$$

where $\mathbf{s}=\left(s_{x}, s_{y}, s_{z}\right)$ and the $N$ coefficients $a_{i}$ are the elements of the vector $\mathbf{a}$. The elements of the $3 \times N$ matrix $\mathbf{M}$ are the values of $s_{i}^{x}$, etc.

\subsubsection{Definition of tip, tilt and defocus}

The inversion of this equation provides the combination of basis modes required to generate the image shifts. As $\mathbf{M}$ is non-square, we can employ pseudo-inversion to give the minimum norm solution

$$
\mathbf{a}=\mathbf{M}^{\dagger} \mathbf{s},
$$

where the three columns of the $N \times 3$ pseudo-inverse matrix $\mathbf{M}^{\dagger}$ are the coefficients required to generate unit image shifts in the $x, y$ and $z$ directions. Equivalently, we defined our three new displacement modes, denoted by $\Lambda_{1}, \Lambda_{2}$ and $\Lambda_{3}$ respectively, in terms of the basis modes $X_{i}$ as

$$
\Lambda_{n}=\sum_{i=1}^{N}\left(\mathbf{M}^{\dagger}\right)_{i, n} X_{i}
$$

where $n=1,2,3$. For this demonstration we chose to correct the first 14 basis modes, which were the most influential. The displacement modes derived from the first 14 basis modes are depicted in Fig. 2(a). As in Fig. 1, these are calculated shapes that approximate the actual shapes produced by the DM.

\subsubsection{Removal of tip, tilt and defocus}

Tip, tilt and defocus components can be removed from the basis modes to provide displacement-free basis modes $X_{i}^{\prime}$ using the following orthogonal projection calculation:

$$
\mathbf{X}^{\prime}=\left(\mathbf{I}-\mathbf{M}^{\dagger} \mathbf{M}\right) \mathbf{X}
$$

(a)
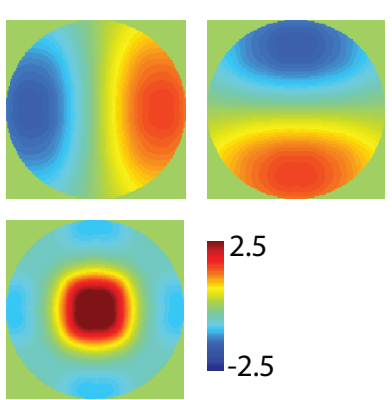

2.5

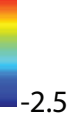

(c)
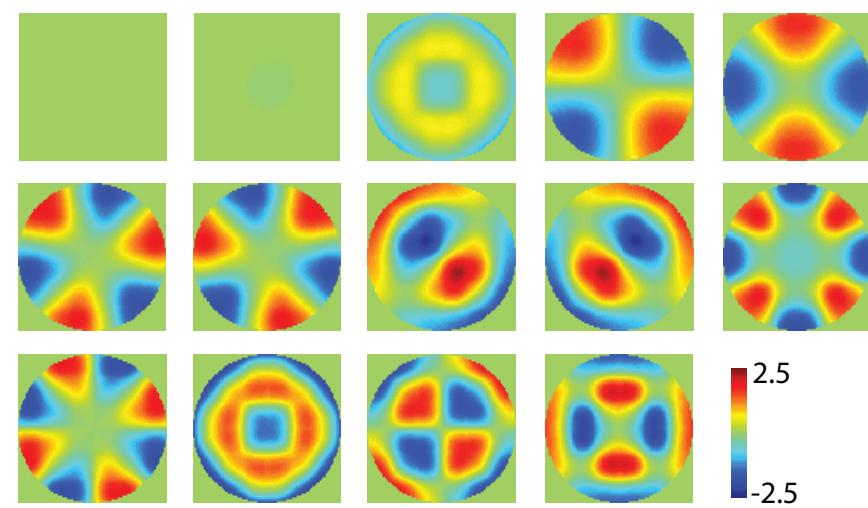

2.5

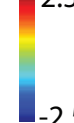

FIG. 2 (a) Plots of the tip, tilt and defocus modes as derived from the first 14 basis modes shown in Fig. 1. (b) The absolute values of the matrix for conversion of basis modes into displacement free modes. (c) Plots of the basis modes after removal of the displacement mode components.

where the elements of the vectors $\mathbf{X}$ and $\mathbf{X}^{\prime}$ are the sets of initial and derived modes, i.e. $\mathbf{X}=\left(X_{1}, \ldots, X_{N}\right)$ and $\mathbf{X}^{\prime}=\left(X_{1}^{\prime}, \ldots, X_{N}^{\prime}\right)$. The matrix $\left(\mathbf{I}-\mathbf{M}^{\dagger} \mathbf{M}\right)$ is the projection matrix that removes the displacement components. The resulting displacement free modes $X_{i}^{\prime}$ are shown in Fig. 2(c). Fig. 2(b) depicts the matrix that converts the basis modes $\mathbf{X}$ to $\mathbf{X}^{\prime}$. The control signals for driving the DM are calculated as

$$
\mathbf{c}=\mathbf{B}\left(\mathbf{I}-\mathbf{M}^{\dagger} \mathbf{M}\right) \mathbf{a}^{\prime}
$$

where the elements of the vector $\mathbf{a}^{\prime}$ are the coefficients of the displacement free modes.

\subsubsection{Orthogonalisation of modes}

Débarre et al. showed that optimal modes could be obtained through measurement of the form of an image quality metric $M$ as a function of the different aberration mode coefficients [3]. We describe here a similar approach to derive new optimal modes $Y_{i}$ from the displacement-free basis set $X_{i}^{\prime}$.

For the purposes of this procedure, we defined the metric function $M$ as the total THG intensity from an axial (x-z) image of a coverglass-air interface. For small aberration amplitudes, the metric takes the approximate paraboloidal form

$$
M \approx q\left(1-\sum_{i} \sum_{j} \alpha_{i j} a_{i} a_{j}\right)
$$

where $q$ is a constant. This can also be expressed conveniently as

$$
M \approx q\left(1-\mathbf{a}^{\mathrm{T}} \mathbf{A} \mathbf{a}\right)
$$




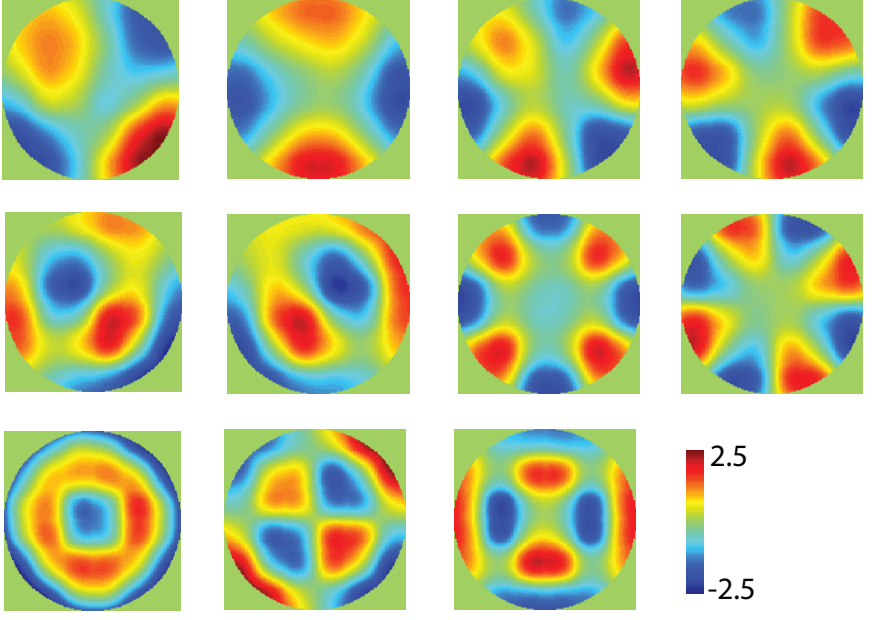

FIG. 3 The optimal aberration modes $Y_{i}$ after the experimental orthogonalisation procedure of the displacement-free modes.

where the elements of the matrix $\mathbf{A}$ are the values of $\alpha_{i j}$. In order to derive the optimal modes, we should first obtain the matrix A.

For each pair of basis modes $X_{i}^{\prime}$ and $X_{j}^{\prime}$ with $i \neq j$, the metric $M$ was measured for a number of test aberrations $\Phi_{(i, j), n}$, which contained certain combinations of the two modes. The mode combinations are chosen to have a constant total magnitude $\gamma$ so that the $n$th test aberration was

$$
\Phi_{(i, j), n}=\gamma\left[\cos \left(\frac{2 \pi}{T} n\right) X_{i}^{\prime}+\sin \left(\frac{2 \pi}{T} n\right) X_{j}^{\prime}\right]
$$

where $T$ is the total number of test aberrations. The constant $\gamma$ was chosen to be small enough such that the assumption of small aberration magnitude was valid. The collection of $T$ metric measurements form a polar plot, where the measured metric values are interpreted as radial coordinates and the numbers $\frac{2 \pi n}{T}$ as angular coordinates. The resulting elliptical plot is a "section" through the multidimensional paraboloidal peak of $M$ that shows the orientation of the paraboloid to the axes.

Several of these plots, acquired for different pairs of basis modes, provide sufficient information to reconstruct $M$ and obtain the optimum modes. The data were fitted to the ellipsoid $\sum_{i} \sum_{j} \alpha_{i j} a_{i} a_{j}=\mathbf{a}^{\mathrm{T}} \mathbf{A a}=c$, where $c$ was a constant, which in turn provided the matrix $\mathbf{A}$. The eigendecomposition of this matrix gives $\mathbf{A}=\mathbf{V D V}^{\mathrm{T}}$, where $\mathbf{V}$ consists of the eigenvectors of $\mathbf{A}$ and the elements of the diagonal matrix $\mathbf{D}$ are the corresponding eigenvalues. Using this decomposition, we can define a new modal expansion for the phase as $\Phi(\mathbf{r})=\sum_{i} b_{i} Y_{i}(\mathbf{r})$. Equation 14 can be rewritten as

$$
M \approx q\left(1-\mathbf{a}^{\mathrm{T}} \mathbf{V D V}^{\mathrm{T}} \mathbf{a}\right)=q\left(1-\mathbf{b}^{\mathrm{T}} \mathbf{b}\right)=q\left(1-\sum_{i=1}^{N} b_{i}^{2}\right)
$$

where the elements of the vector $\mathbf{b}=\mathbf{D}^{1 / 2} \mathbf{V}^{\mathrm{T}} \mathbf{a}$ are the new modal coefficients $b_{i}$. This representation of $M$ now has the same desired form as Eq. 4. This shows that the new set of modes has the optimal properties that permit the independent optimisation of each mode. The optimum modes $Y_{i}(\mathbf{r})$ (a)
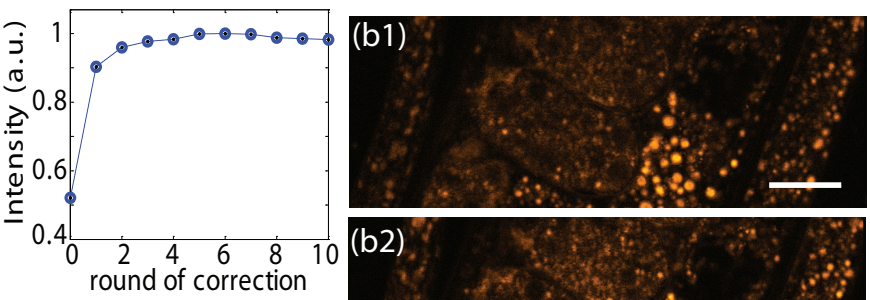

(c)

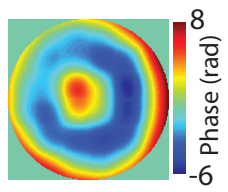

(d)

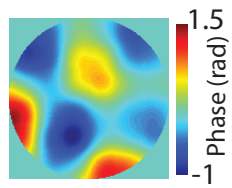

(b2)
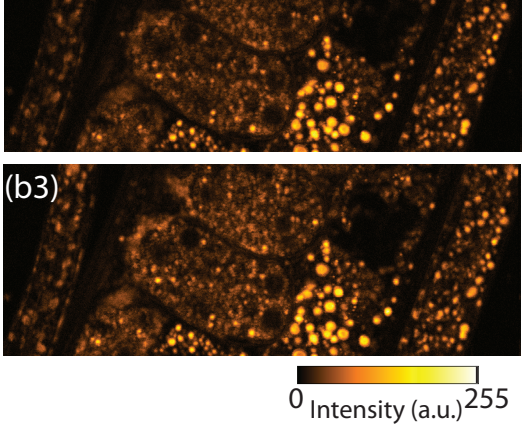

FIC. 4 Correction of aberrations using the displacement-free optimal modes. (a) The image intensity metric for subsequently applied correction cycles. (b1-b3) THG images of $C$. Elegans specimen section, $25 \mu \mathrm{m}$ deep in the sample before correction, after correcting system aberrations and after further correction of specimen aberrations, respectively. Scale bar is $10 \mu \mathrm{m}$. (c) Correction phase applied for compensating system induced aberrations and (d) the additional correction for specimen aberrations.

can then be calculated as

$$
Y_{i}(\mathbf{r})=\sum_{j} \frac{V_{i j}}{\sqrt{D_{j j}}} X_{j}^{\prime}(\mathbf{r})
$$

where $V_{i j}$ and $D_{j j}$ are elements of $\mathbf{V}$ and $\mathbf{D}$, respectively.

The DM control signals for generating the aberration $\Phi(\mathbf{r})=\sum_{i} b_{i} Y_{i}(\mathbf{r})$ can be calculated as

$$
\mathbf{c}=\mathbf{B}\left(\mathbf{I}-\mathbf{M}^{\dagger} \mathbf{M}\right) \mathbf{V D}^{-1 / 2} \mathbf{b}
$$

Figure 3 shows the resulting optimal modes, as defined by Eq. 17. Only the 11 most significant modes are shown in this figure. The remaining three modes are degenerate, due to the previous removal of the three displacement modes.

\section{Aberration correction using optimal modes}

The effectiveness of the displacement-free optimal modes was verified by implementing the sensorless aberration correction scheme in the THG microscope described in Section 3.2. Figure 4 shows the results of this correction procedure when applied to a C. Elegans specimen. The C. Elegans was maintained in between two coverglass on nematode growth medium agar. Firstly, the system induced aberrations were corrected using THG signal from the coverglass-medium interface directly beneath the $C$. Elegans. Only $N=11$ modes shown in Figure 3 were included in the correction procedure. Trial aberrations of bias amplitudes $b= \pm 0.5$ were applied in a sequential manner measuring the corresponding quality metric, which was chosen to be the total image intensity [11]. The final correction 
(a)

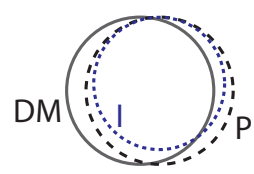

(c)

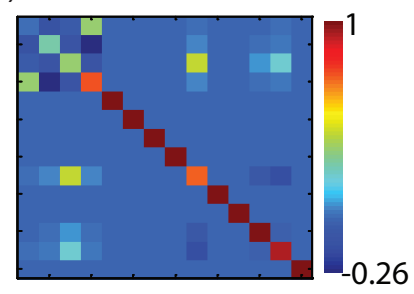

(b)
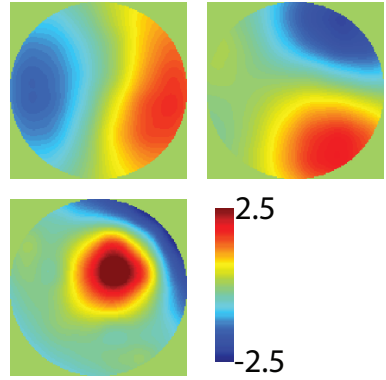

FIG. 5 Displacement modes of a misaligned system. (a) Schematic showing the approximate misalignment introduced to the system. P: objective pupil, DM: deformable mirror active area, I: iris. (b) The tip, tilt and defocus displacement modes. (c) The conversion matrix for generating displacement-free modes from the first 14 basis modes. The plots show simulations of the whole DM aperture. The functional aperture corresponds to that in part (a) of this figure.

phase was then estimated as described in section 2.4. Rapid acquisition of low resolution images was sufficient to estimate the correction phase and one correction cycle took approximately 1 minute. In Figure 4 part (a) shows the value of the image intensity metric after each repeated correction cycle applied to the coverglass-medium interface. The metric reaches $90 \%$ of the maximum achieved value after the first cycle. From the second cycle onwards, the metric is greater than $96 \%$. Figure 4(b) shows images of a lateral section , $25 \mu \mathrm{m}$ deep in the C. Elegans specimen taken before and after aberration correction. Part (b1) was taken without correction. Part (b2) was after correction of system aberrations. This alone improved the image intensity by $32.5 \%$. Part (b3) was after further correction of specimen induced aberrations. This allowed an additional $11 \%$ improvement of the image intensity. Figure 4(c) shows the correction phase used for the system aberrations and Fig. 4(d) is the additional correction for specimen aberrations.

\section{Robustness of method}

As the modal derivation was based entirely upon empirical measurement, rather than off-line calculation, the scheme was shown to be robust with respect to changes in experimental conditions. In particular, any misalignments of the system were automatically taken into account by the calibration methods. This is in contrast to off-line methods where alignment errors can lead to significant differences between theoretical predictions and practical performance. In order to illustrate this robustness, we repeated the procedure for aberration mode derivation after intentionally misaligning the system. The pupils of the DM and objective lens were offset as shown in Figure 5(a). An iris was also used to reduce the size of the functional pupil. The displacement modes were determined using the method described above. The resulting DM shapes are shown in Figure 5(b). Note that these represent the original DM active area, whereas the new displacement modes would be defined only over the misaligned pupil shown in

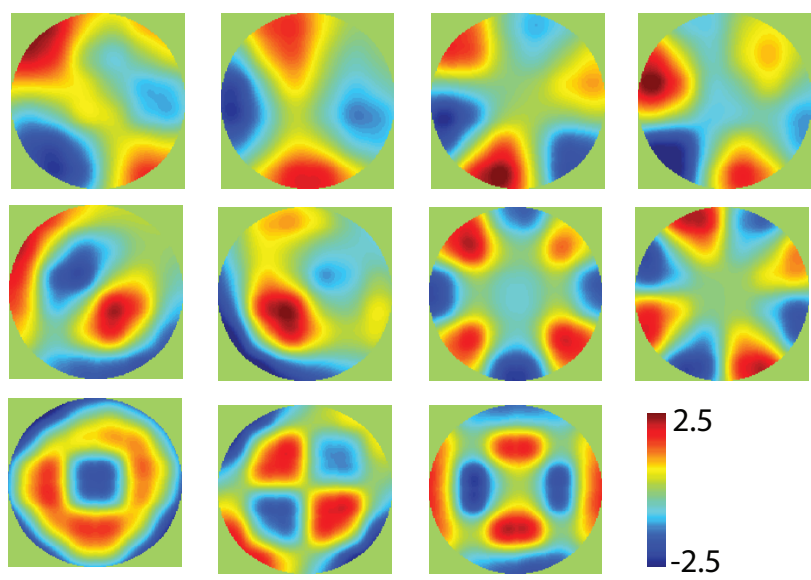

FIG. 6 The optimum aberration modes derived for the misaligned experimental system. The plots show a simulation of the whole DM aperture. The functional aperture corresponds to that in the diagram of Figure $5(\mathrm{a})$.

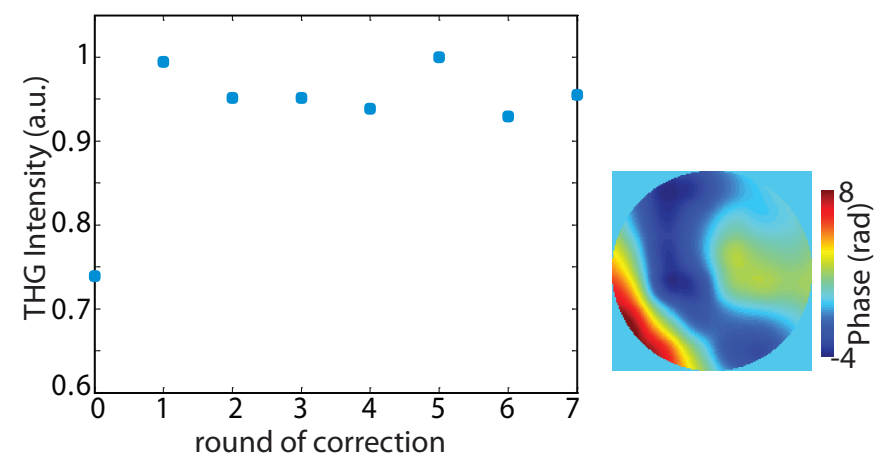

FIG. 7 THG image intensity as a function of the number of iterations of the correction procedure and the corresponding correction phase.

Figure 5(a). These new displacement modes were removed from the basis set and the conversion matrix for the generation of these displacement free modes from the basis modes is depicted in Figure 5(c). The experimental orthogonalisation procedure was followed in order to derive the optimal modes for this optical configuration. The resulting modes are shown in Figure 6. Again, these plots show the original active DM area; parts of the illustrated plots therefore lie outside of the system pupil that was actually used for the calibration. As should be expected, these results are different to those obtained previously (Figure 3) using the full, original pupil.

The THG signal from a coverglass-air interface was used to verify the effectiveness of these aberration modes in the modal correction scheme. Figure 7 shows the variation of image intensity for successive rounds of correction and the correction phase estimated. The optimum correction was achieved after the first cycle. As expected, the correction quality was lower than the case presented in the Figure 4 . This is because a smaller proportion of the DM was used in the misaligned system. 


\section{Discussion}

The method presented in this paper permits the calibration of an adaptive optical microscope without the need for an additional wavefront sensor or interferometer to characterise the adaptive element. This considerably reduces the complexity of the optical systems of AO microscopes, as the calibration method uses exactly the same hardware as the adaptive imaging system itself. In contrast to previous calibration methods, all measurements, including the important step of the removal of displacement components from the basis modes, are based upon image phenomena.

In contrast to off-line calculation methods, the new procedure is robust in the presence of practical variations, as illustrated by the derivation of optimal modes for the system with misaligned pupils. It is also able to cope with other non-ideal configurations, such as errors in magnification between the DM and the objective pupil, or spatial variations in beam profile, for example due to apodisation effects or due to the laser beam profile when using an underfilled pupil. The calibration can also be carried out in the same microscope system for multiple objective lenses, which typically have different pupil sizes and hence use a different region of the DM for aberration correction. Since the re-imaging stage can compensate for the effects of mismatch between the pupil size and DM actuators, the new procedure would also permit quicker re-calibration if the objective lenses needed to be changed (tens of minutes, but this could be shorter with optimisation for speed).

Whilst the sensorless nature of this method is in many ways advantageous, it does have the drawback that there is no absolute reference for the aberration measurements. This means it is only possible to estimate the form and magnitude of the correction aberration using an approximate model of the DM. A further consequence is that this method does not permit training of the DM system to produce specific aberration modes, only sets of modes with the desired mutually orthogonal properties. AO systems employing spatial light modulators do not generally require the measurement of the influence functions. However, a careful alignment of the phase patterns on these elements is also necessary for the correct operation of the AO system. In such systems, an approach similar to that described above could be used. Although we have demonstrated the operation of this method in a THG microscope, we emphasise that this method is applicable to any adaptive microscope system including confocal, multiphoton and widefield microscopes, as long as a suitable optimisation metric is chosen.

\section{References}

[1] M. J. Booth, "Adaptive optics in microscopy", Philos. Transact. A Math. Phys. Eng. Sci. 365 2829-2843 (2007).

[2] D. Débarre, E. J. Botcherby, T. Watanabe, S. Srinivas, M. J. Booth, and T. Wilson, "Image-based adaptive optics for two-photon microscopy", Opt. Lett. 34 2495-2497 (2009).

[3] D. Débarre, E. J. Botcherby, M. J. Booth, and T. Wilson, "Adaptive optics for structured illumination microscopy.", Opt. Express. 16 9290-9305 (2008).

[4] M. J. Booth, "Wave front sensor-less adaptive optics: a modelbased approach using sphere packings", Opt. Express 14 13391352 (2006).

[5] M. J. Booth, "Wavefront sensorless adaptive optics for large aberrations", Opt. Lett. 32 5-7 (2007).

[6] D. Débarre, M. J. Booth, and T. Wilson, "Image based adaptive optics through optimisation of low spatial frequencies", Opt. Express 15 8176-8190 (2007).

[7] N. Olivier, D. Dèbarre, and E. Beaurepaire, "Dynamic aberration correction for multiharmonic microscopy.", Opt. Lett. 34 3145-3147 (2009).

[8] A. Jesacher, A. Thayil, K. Grieve, D. Dèbarre, T. Watanabe, T. Wilson, S. Srinivas, and M. Booth, "Adaptive harmonic generation microscopy of mammalian embryos.", Opt. Lett. 34 3154-3156 (2009).

[9] R. P. Grosso and M. Yellin, “Membrane Mirror As An Adaptive Optical Element", J. Opt. Soc. Am. 67 399-406 (1977).

[10] C. Paterson, I. Munro, and J. C. Dainty, "A low cost adaptive optics system using a membrane mirror", Opt. Express 6 175-185 (2000).

[11] A. Thayil, A. Jesacher, T. Wilson, and M. J. Booth, "Influence of aberrations in third harmonic generation microscopy", J. Opt. 12 084009 (2010).

\section{Acknowledgements}

This work was funded by grants from the Biotechnology and Biological Sciences Research Council, United Kingdom (grant number BB/F011512/1) and the Engineering and Physical Sciences Research Council, United Kingdom (grant number $\mathrm{EP} / \mathrm{E} 055818 / 1)$. 